\title{
EFFECT OF PRESSURE ON MAGNETIC STATES OF EASY-PLANE ANTIFERROMAGNETS
}

\author{
Yu.N. Mitsay, Yu.A. Fridman, G.A. Bairamalieva, C.N. Alexeev \\ M.V. Frunze Simferopol State University, Yaltinskaya st., 4, Simferopol, Ukraine
}

AND M.S. KOCHMAŃSKI

Institute of Physics, Pedagogical University, Rejtana 16 A, 35-310 Rzcszów, Poland

(Received January 8, 1997; in final form March 3, 1997)

The phase $H-P-\beta$ diagram of an easy-plane strongly anisotropic antiferromagnet is studied. It is shown that in such a system a realization of the phase with the nonmagnetic order parameter, the so-called quadruple phase, is possible. The realization of this phase is caused by several purely quantum effects, moreover the mechanism of its appearance differs in principle from that of the antiferromagnet or ferromagnet. The possibility of the disappearance of the angular phase (the so-called quadruple ferromagnetic phase) is also shown.

PACS numbers: $75.50 . \mathrm{Ee}, 75.30 . \mathrm{Kz}$

\section{Introduction}

Easy-plane antiferromagnets are traditional objects of investigation [1]. An interest in the investigation of such systems comes from the fact that their dynamics is determined by the Goldstein spectrum of elementary excitations and their magnetostriction properties are enhanced by the exchange interaction.

Recently, low-temperature antiferromagnets such as $\mathrm{NiSiF}_{6} \cdot 6 \mathrm{H}_{2} \mathrm{O}$, $\mathrm{NiZrF}_{6} \cdot 6 \mathrm{II}_{2} \mathrm{O}$ and other compounds have been studied. The behaviour of such systems in magnetic fields perpendicular to an easy-plane is studied in Refs. [2, 3].

The behaviour of such systems in a longitudinal magnetic field is poorly recognized. In this paper possible magnetic states of the investigated system in a longitudinal magnetic field and under external pressure are determined. A study of magnetic states of magnetically ordered substances under external pressure is an effective method of investigation of such systems since external pressure effectively manifests itself as a magnetic anisotropy.

\section{The formalism and results}

The system under investigation is a strongly anisotropic $(\beta \geq J)$ antiferromagnet (AFM) with the Dzyaloshinskiy exchange. The resulting magnetic momen- 
tum lies in the easy plane, in the same plane $(X O Y)$ in which the applied external magnetic field $H$ lies. We study possible magnetic states in this system under external pressure $P$. Further we assume that external pressure is also applied in the easy plane $(P<0)$. As we demonstrate further it is the most interesting case.

The Hamiltonian of our system has the form

$$
\begin{aligned}
\mathcal{H}= & -H \sum_{n_{i}} S_{n_{i}}^{x}+\frac{\beta}{2} \sum_{n_{i}}\left(S_{n_{i}}^{z}\right)^{2} \\
& +\sum_{n_{1}, n_{2}}\left\{J\left(n_{1}-n_{2}\right) \bar{S}_{n_{1}} \bar{S}_{n_{2}}-D\left(n_{1}-n_{2}\right)\left[\bar{S}_{n_{1}} \times \bar{S}_{n_{2}}\right]_{z}\right\} \\
& +\nu \sum_{n_{i}} S_{n_{i}}^{j} S_{n_{i}}^{k} U_{j k}\left(n_{i}\right) \\
& +\int \mathrm{d} r\left\{\frac{\lambda+\eta}{2}\left(U_{x x}^{2}+U_{y y}^{2}+U_{z z}^{2}\right)+\eta\left(U_{z y}^{2}+U_{x z}^{2}+U_{y x}^{2}\right)\right. \\
& \left.+\lambda\left(U_{x x} U_{y y}+U_{x x} U_{z z}+U_{y y} U_{z z}\right)+P U_{x x}\right\}
\end{aligned}
$$

where $S_{n_{i}}^{\alpha}-$ spin operator in a site of sublattice $i, D\left(n_{1}-n_{2}\right)<0$ - the Dzyaloshinskiy exchange, $\nu$ - constant of magnetoelastic exchange, $\lambda, \eta$ - elasticity modules, $U_{j, k}\left(n_{i}\right)$ - tensor of deformations. In Eq. (1) the first three terms describe the magnetic subsystem, the fifth term describes the elastic one and the fourth term - magnetoelastic interaction. We shall carry out further investigations in a low-temperature limit: $T \ll T_{\mathrm{N}}, T_{\mathrm{N}}-$ Néel's temperature, since in this case it is possible to make all calculations analytically. Besides, without the loss of generality we assume that a magnetic ion spin $S=1, M=M_{1}+M_{2}$.

The orientation of magnetic momenta of sublattices for the system described by (1) is shown in Fig. 1. Let us rotate coordinate system around the $O Z$ axis

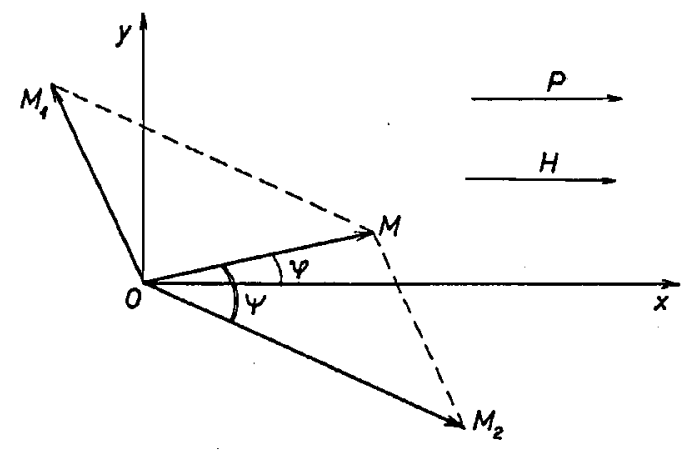

Fig. 1. The orientation of the magnetic momenta of sublattices. 
(perpendicular to the plane of Fig. 1) so that the new axis of quantization $\xi_{i}$ became parallel to the vector of magnetization of the $i$-th sublattice. In this local coordinate system we introduce new operators related to the previous ones by the equations

$$
\begin{aligned}
& S_{n_{i}}^{x}=S_{n_{i}}^{\xi} \cos \theta_{i}+(-1)^{i+1} S_{n_{i}}^{\eta} \sin \theta_{i}, \\
& S_{n_{i}}^{y}=(-1)^{i+1} S_{n_{i}}^{\xi} \sin \theta_{i}+S_{n_{i}}^{\eta} \cos \theta_{i}, \\
& S_{n_{i}}^{z}=S_{n_{i}}^{\zeta}, \\
& \psi=\frac{\theta_{1}+\theta_{2}}{2}, \quad \varphi=\frac{\theta_{1}-\theta_{2}}{2} .
\end{aligned}
$$

We shall carry out further calculations with the use of the Hubbard operators $[4,5]$ which permit exact account of one-ion and magnetoelastic exchanges. These operators are built on eigenfunctions of the one-site Ilamiltonian $\mathcal{H}_{0}$. After separation of the mean field in the exchange part of (1) and transition to local coordinates (2) we obtain for the one-site Hamiltonian

$$
\begin{aligned}
\mathcal{H}_{0}= & -\sum_{n_{i}} H_{i} S_{n_{i}}^{\xi}+\sum_{n_{i}}(-1)^{i+1} \bar{H}_{i}\left(S_{n_{i}}^{+}+S_{n_{i}}^{-}\right. \\
& -\frac{\beta}{8} \sum_{n_{i}}\left\{\left(S_{n_{i}}^{+}\right)^{2}+\left(S_{n_{i}}^{-}\right)^{2}-S_{n_{i}}^{+} S_{n_{i}}^{-}-S_{n_{i}}^{-} S_{n_{i}}^{+}\right\} \\
& +\frac{\nu}{2} \sum_{n_{i}}\left\{\left(U_{x x}^{i}+U_{y y}^{i}-U_{z z}^{i}-A_{i}+B_{i}\right)\left(S_{n_{i}}^{+}\right)^{2}+2 A_{i}\left(S_{n_{i}}^{\xi}\right)^{2}\right. \\
& +(1 / 2)\left(U_{x x}^{i}+U_{y y}^{i}+U_{z z}^{i}-A_{i}\right)\left(S_{n_{i}}^{+} S_{n_{i}}^{-}+S_{n_{i}}^{-} S_{n_{i}}^{+}\right) \\
& \left.+\left(C_{1}^{i}+C_{2}^{i}\right)\left(S_{n_{i}}^{+} S_{n_{i}}^{\xi}+S_{n_{i}}^{\xi} S_{n_{i}}^{+}\right)+\text {h.c. }\right\} .
\end{aligned}
$$

Here we denote

$$
\begin{aligned}
& S_{n_{i}}^{ \pm}=S_{n_{i}}^{\eta} \pm \mathrm{i} S_{n_{i}}^{\zeta}, \quad\left\langle S_{n_{1}}^{\xi}\right\rangle=\left\langle S_{n_{2}}^{\xi}\right\rangle=\left\langle S^{\xi}\right\rangle, \\
& H_{i}=H \cos \theta_{i}-\left\langle S^{\xi}\right\rangle\left(J_{0} \cos 2 \psi+D_{0} \sin 2 \psi\right), \\
& \bar{H}_{i}=\frac{1}{2}\left[H \sin \theta_{i}-\left\langle S^{\xi}\right\rangle\left(J_{0} \sin 2 \psi+D_{0} \cos 2 \psi\right)\right], \\
& A_{i}=U_{x x}^{i} \cos ^{2} \theta_{i}+U_{y y}^{i} \sin ^{2} \theta_{i}+(-1)^{i+1} U_{x y}^{i} \sin 2 \theta_{i}, \\
& B_{i}=-2 \mathrm{i}\left[U_{y z}^{i} \cos \theta_{i}+(-1)^{i+1} U_{x z}^{i} \sin \theta_{i}\right], \\
& C_{1}^{i}=(-1)^{i}\left(U_{x x}^{i}-U_{y y}^{i}\right) \sin 2 \theta_{i}+2 U_{x y}^{i} \cos 2 \theta_{i}, \\
& C_{2}^{i}=-2 \mathrm{i}\left[U_{x z}^{i} \cos \theta_{i}+(-1)^{i+1} U_{y z}^{i} \sin \theta_{i}\right],
\end{aligned}
$$

where $J_{0}=\sum_{n} J(n)$. Solving the one-ion problem

$$
\mathcal{H}_{0}\left(n_{i}\right) \Psi_{n_{i}}(M)=E_{M} \Psi_{n_{i}}(M),
$$


with the Hamiltonian (3) we determine eigenvalues and eigenfunctions of $\mathcal{H}_{0}$ $(M$ - magnetic quantum numbers; for $S=1, M=1,0,-1)$

$$
\begin{aligned}
& E_{1,-1}^{i}=\frac{\beta}{4}+\frac{\nu / 2}{H_{i}^{2}+4 \bar{H}_{i}^{2}}\left\{\left(U_{x x}^{i}+U_{y y}^{i}\right)\left(H_{i}^{2}+8 \bar{H}_{i}^{2}\right)+U_{z z}^{i}\left(H_{i}^{2}+4 \bar{H}_{i}^{2}\right)\right. \\
& \left.+A_{i}\left(H_{i}^{2}-4 \bar{H}_{i}^{2}\right)+(-1)^{i+1} 2 C_{1}^{i} H_{i} \bar{H}_{i}\right\} \mp\left\{\frac{\beta^{2}}{16}+H_{i}^{2}+4 \bar{H}_{i}^{2}-\frac{\nu \beta / 4}{H_{i}^{2}+4 \bar{H}_{i}^{2}}\right. \\
& \times\left[\left(U_{x x}^{i}+U_{y y}^{i}\right) H_{i}^{2}-U_{z z}^{i}\left(H_{i}^{2}+4 \bar{H}_{i}^{2}\right)-A_{i}\left(H_{i}^{2}-4 \bar{H}_{i}^{2}\right)+(-1)^{i+1} 2 C_{1}^{i} H_{i} \bar{H}_{i}\right] \\
& \quad+\left(\frac{\nu / 2}{H_{i}^{2}+4 \bar{H}_{i}^{2}}\right)^{2}\left[\left(U_{x x}^{i}+U_{y y}^{i}\right) H_{i}^{2}-U_{z z}^{i}\left(H_{i}^{2}+4 \bar{H}_{i}^{2}\right)\right. \\
& \left.\left.\quad-A_{i}\left(H_{i}^{2}-4 \bar{H}_{i}^{2}\right)+(-1)^{i+1} 2 C_{1}^{i} H_{i} \bar{H}_{i}\right]^{2}\right\}^{1 / 2}, \\
& E_{0}^{i}=\frac{\beta}{2}+\frac{\nu}{H_{i}^{2}+4 \bar{H}_{i}^{2}}\left\{\left(U_{x x}^{i}+U_{y y}^{i}\right) H_{i}^{2}+U_{z z}^{i}\left(H_{i}^{2}+4 \bar{H}_{i}^{2}\right)-A_{i}\left(H_{i}^{2}-4 \bar{H}_{i}^{2}\right)\right. \\
& \left.\quad+(-1)^{i+1} 2 C_{1}^{i} H_{i} \bar{H}_{i}\right\} .
\end{aligned}
$$

The Hubbard operators are built on eigenfunctions of $\mathcal{H}_{0}$ by standard rules [6], $Y_{n_{i}}^{M^{\prime} M}=\left|\Psi_{n_{i}}\left(M^{\prime}\right)\right\rangle\left\langle\Psi_{n_{i}}(M)\right|$ and describe the transition of a magnetic ion from a state $M$ to a state $M^{\prime}$. These operators are related to the spin ones by the well-known equations [7].

From the condition of a free energy density minimum

$\mathcal{F}=\mathcal{F}_{0}-T \ln \mathcal{Z}$,

where $\mathcal{F}_{0}-$ a free energy of the elastic subsystem, determined by the last term in (1). Let us determine equilibrium (spontaneous) deformations $U_{j k}^{(0)}\left(n_{i}\right)$ :

$$
\begin{aligned}
U_{x x}^{(0) i} & =\frac{b_{1}^{i}(\eta+2 \lambda)-\lambda\left(b_{2}^{i}-\lambda b_{3}^{i}\right)}{\eta(\eta+3 \lambda)}, \quad U_{y y}^{(0) i}=\frac{b_{2}^{i}(\eta+2 \lambda)-\lambda\left(b_{1}^{i}+b_{3}^{i}\right)}{\eta(\eta+3 \lambda)} \\
U_{z z}^{(0) i} & =\frac{b_{3}^{i}(\eta+2 \lambda)-\lambda\left(b_{1}^{i}+b_{2}^{i}\right)}{\eta(\eta+3 \lambda)}, \quad U_{x z}^{(0) i}=U_{y z}^{(0) i}=0 \\
U_{x y}^{(0) i} & =\frac{(-1)^{i+1} \nu}{2 \eta} \frac{\left(H_{i}^{2}-4 \bar{H}_{i}^{2}\right) \sin 2\left[\psi+(-1)^{i+1} \varphi\right]}{H_{i}^{2}+4 \bar{H}_{i}^{2}} \\
& -\frac{4 H_{i} \bar{H}_{i} \cos 2\left[\psi+(-1)^{i+1} \varphi\right]}{\left(H_{i}^{2}+4 \bar{H}_{i}^{2}\right) z_{i}^{(0)}} \\
& \times\left\{\cosh \frac{\kappa_{i}^{0}}{T}+\frac{\beta}{4 \kappa_{i}^{0}} \sinh \frac{\kappa_{i}^{0}}{T}-\exp (-\beta / 4 T)\right\},
\end{aligned}
$$


where

$$
\begin{aligned}
& b_{1}^{i}=-P \\
&-\frac{\nu}{z_{i}^{(0)}}\left\{\left[1+\frac{\left(H_{i} \cos \left(\psi+(-1)^{i+1} \varphi\right)+2 \bar{H}_{i} \sin \left(\psi+(-1)^{i+1} \varphi\right)\right)^{2}}{H_{i}^{2}+4 \bar{H}_{i}^{2}}\right] \cosh \frac{\kappa_{i}^{0}}{T}\right. \\
&-\frac{\left(H_{i} \sin \left(\psi+(-1)^{i+1} \varphi\right)-2 \bar{H}_{i} \cos \left(\psi+(-1)^{i+1} \varphi\right)\right)^{2}}{H_{i}^{2}+4 \bar{H}_{i}^{2}} \\
&\left.\times\left[\frac{\beta}{4 \kappa_{i}^{0}} \sinh \frac{\kappa_{i}^{0}}{T}-\exp (-\beta / 4 T)\right]\right\} \\
& b_{2}^{i}= \frac{\nu}{z_{i}^{(0)}}\left\{\left[1+\frac{\left(H_{i} \sin \left(\psi+(-1)^{i+1} \varphi\right)+2 \bar{H}_{i} \cos \left(\psi+(-1)^{i+1} \varphi\right)\right)^{2}}{H_{i}^{2}+4 \bar{H}_{i}^{2}}\right] \cosh \frac{\kappa_{i}^{0}}{T}\right. \\
&-\frac{\left(H_{i} \cos \left(\psi+(-1)^{i+1} \varphi\right)-2 \bar{H}_{i} \sin \left(\psi+(-1)^{i+1} \varphi\right)\right)^{2}}{H_{i}^{2}+4 \bar{H}_{i}^{2}} \\
& z_{i}^{(0)}= 2 \cosh \frac{\kappa_{i}^{0}}{T}+\exp \left(-\frac{\beta}{4 T}\right) \cdot \\
& b_{3}^{i}=-\frac{\nu}{z_{i}^{(0)}}\left[\cosh \frac{\kappa_{i}^{0}}{T}+\frac{\beta}{4 \kappa_{i}^{0}} \sinh \frac{\kappa_{i}^{0}}{T}-\exp (-\beta / 4 T)\right] \\
&\left.\left.4 \kappa_{i}^{0} \sinh \frac{\kappa_{i}^{0}}{T}-\exp (-\beta / 4 T)\right]\right\}
\end{aligned}
$$

As it is evident from relationships (4) and (6) an energy level corresponding to the ground state is $E_{1}^{i}$. The analytical expression for it in our approximation is

$$
\begin{aligned}
E_{1}^{i}= & -\frac{H_{i}^{2}+4 \bar{H}_{i}^{2}}{\beta}-\frac{\nu P}{\eta}+\frac{2 \nu P}{\eta} \frac{H_{i} \bar{H}_{i}}{H_{i}^{2}+4 \bar{H}_{i}^{2}} \sin \left\{2\left[\psi+(-1)^{i+1} \varphi\right]\right\} \\
& +\mathrm{O}\left(\frac{\nu^{2}}{\eta}\right) .
\end{aligned}
$$

In this case is possible to represent the density of free energy in the form

$$
\mathcal{F}^{i}=\mathcal{F}_{0}^{i}+E_{1}^{i}
$$

where $\mathcal{F}_{0}^{i}$ is the density of free energy of the elastic subsystem. With the use of Eq. (9) we determined possible phases of the investigated system. We assume that the external magnetic field $H$ and pressure $P$ are such that mean magnetization at sites is oriented as shown in Fig. 1.

When the value of a magnetic field is larger than $H_{\mathrm{c}_{2}}$ (at constant pressure) the mean magnetization vector is oriented along the direction of a magnetic 
field $H$. In this case the angle $\varphi=0$, while $\psi$ is determined from the condition of a minimum of a free energy density $\mathcal{F}^{i},(9)$. From this condition in the phase where $\varphi=0, H_{i}=0$ one can obtain the following relationship:

$$
\cos \psi=\frac{H+\left\langle S^{\xi}\right\rangle\left|D_{0}\right|}{2 J_{0}\left\langle S^{\xi}\right\rangle} .
$$

One can demonstrate that in the vicinity of a field $H_{\mathrm{c}_{2}}$, mean magnetization of sublattice approximately equals unity, $\left\langle S^{\xi}\right\rangle \approx 1$. Let us call the phase occurring in fields $H \geq H_{c_{2}}$ the FM1-phase of magnetoelastic waves. We determine the value of $H_{c_{2}}$ from the condition of vanishing of a coefficient at the quadratic in $\varphi$ term in the expansion of a free energy (9):

$$
H_{\mathrm{c}_{2}}=-\frac{\left|D_{0}\right|}{2}+\sqrt{\frac{D_{0}^{2}}{4}-2 d_{0} J_{0}},
$$

where $d_{0}=P \nu / \eta$. Further increase in the field leads not only to the fact that $M$ is parallel to the field but that vectors of sublattice magnetizations orient themselves along field as well (i.e. $\psi=\varphi=0$ ). This configuration realizes at $H \geq H_{\mathrm{c}_{3}}$, where

$$
H_{\mathrm{c}_{3}}=2 J_{0}-\left|D_{0}\right| \text {. }
$$

Let us call the phase which realizes at $H \geq H_{\mathrm{c}_{3}}$ the FM2-phase.

The most interesting is the case of small fields at which the so-called quadruple (QU) phase is realized. For this phase the equality to zero of a magnetic vector order parameter is characteristic [8]. For our system it means that the mean sublattice magnetization equals zero as well as the mean magnetization at the site. Besides it is well known $[9,8]$ that for easy-plane ferromagnets in a transversal magnetic field the realization of the QU-phase is connected with the inversion of energy levels, i.e. $E_{0}$ becomes the lowest level in this phase. In the case of our system there is no such inversion and the realization of the QU-phase is connected with the exhibition of some purely quantum effects.

Let us consider this question in detail. Let us suppose that at certain field $H \leq H_{\mathrm{c}_{1}}$ the system is transformed into the QU-phase. Let us call the magnetic phase realizing at fields $H_{\mathrm{c}_{1}} \leq H \leq H_{\mathrm{c}_{2}}$ the QU-phase (quadruple ferromagnetic). In our problem pressure plays the role of an "effective" anisotropy with the axis of the easy magnetization parallel to $O Y$ axis [10]. For fields in the vicinity of the QFM-QU phase transition a minimum of the free energy density (9) corresponds to values of $\varphi$ and $\psi$ close to $\varphi=\pi / 2, \psi=0$, i.e. the vector of the mean magnetization of a system as well as vectors of the mean sublattice magnetization tend to "turn" to an "effective" axis of the magnetization.

The mean magnetization of sublattices equals

$$
\left\langle S_{n_{i}}^{\xi}\right\rangle=4 \frac{H}{\beta} \cos (\psi+\varphi) .
$$

Typical antiferromagnets with the Dzyaloshinskiy exchange are characterised by the values of angles $(\psi \approx \pi / 2)$. A sharp decrease in this angle to small values in our case is caused as it is evident from Eq. (10), by the sublattice magnetization modulus tending to zero. A similar effect near a critical temperature for $\mathrm{La}_{2} \mathrm{CuO}_{4}$ is discussed in [11]. 
At $H=0$ and $P=0$ and certain values of the system the latter exists in the QU-phase. One can understand this result in such a way: there is no singled out axis in the system, all the directions in the basic plane $(X O Y)$ are equal. Therefore magnetization in different elementary magnetic cells can be directed arbitrarily and an "average" magnetization of a crystal equals zero. This situation is analogous to the realization of the QU-phase in a strongly anisotropic easy-plane ferromagnet [5].

As evident, the ground state of an AFM at $P=0$ and $H=0$ coincides with eigenvector $|1\rangle$ of operator $S^{\xi}, \Psi_{n_{i}}(1)=|1\rangle$. With increasing pressure it is more favourable for mean magnetization to turn along the axis of an "effective" anisotropy $(O Y)$. In this case the ground state of an AFM is a superposition of eigenvectors $|1\rangle$ and $|-1\rangle$ and operator $S^{\xi}, \Psi_{n_{i}}(1)=\cos \delta_{i}|1\rangle+\sin \delta_{i}|-1\rangle$, such a superposition of vectors $|1\rangle$ and $|-1\rangle$ gives rise to the "quantum reduction of a spin" [3]. This effect is intrinsic in easy-axis magnets in a magnetic field perpendicular to an easy axis. In our case the cause of this effect is the presence of the external pressure which plays the role of one-axis anisotropy. It turns out that the QU-phase exists up to pressures determined by the formula

$$
\frac{\nu P_{\mathrm{c}}}{\eta}=\frac{\beta}{2}-J_{0}-2 a_{0}
$$

where $a_{0}=\nu^{2} / 2 \eta$. This formula (12) is obtained from the investigation of the area of existence of a phase with non-zero mean magnetization. The phase which realizes at $H=0$ is shown in Fig. 2 in the $(P, \beta)$ plane.

The field of the QFM-QU phase transition $H_{c_{1}}$ is determined by the standard mean field theory which gives

$$
H_{\mathrm{c}_{1}}=\sqrt{\frac{\beta}{2}\left(\frac{\beta}{2}-d_{0}-J_{0}-2 a_{0}\right)} .
$$

The dependence of angles and sublattice magnetization on the external magnetic field is shown in Figs. 3, 4

$$
\psi_{0}=\frac{D_{0}}{4 J_{0}}+\sqrt{\frac{D_{0}^{2}}{16 J_{0}^{2}}+\frac{d_{0}}{2 J_{0}}} .
$$

Figures $5 \mathrm{a}$ and $5 \mathrm{~b}$ show a phase diagram of the magnet in study in the coordinates $(P, \beta, H)$. As evident from Fig. $5 \mathrm{~b}$ in a $(P, H)$ plane the QFM-FM1 phase transitions as well as the FM1-FM2 ones are possible. However, at a pressure $P_{0}$ :

$$
\tilde{d}_{0}=\frac{P_{0} \nu}{\eta}=\left|D_{0}\right|-2 J_{0},
$$

the QFM-FM2 phase transition is possible, i.e. the FM1 vanishes (Fig. 5b).

In the $(\beta, H)$ plane as follows from Fig. 5a the following transitions are possible: the QU-QFM, QU-QFM1 as well as FM1-FM2. In this case there is also possible the situation when the FM1 phase vanishes, i.e. the QU-FM2 transition is possible at value of an anisotropy constant (Fig. 5a): 


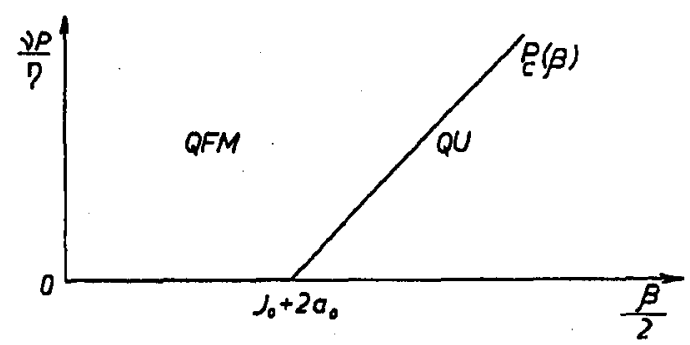

Fig. 2. The phase diagram of an easy-plane AFM in a plane $(P, \beta)$.

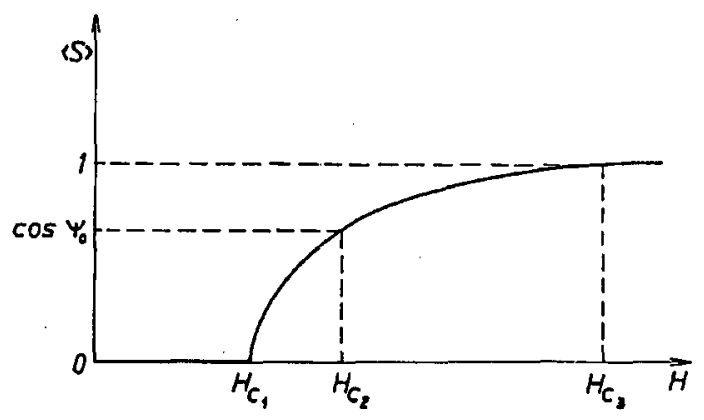

Fig. 3. The dependence of mean sublatlice magnetization on the value of the external magnetic field.
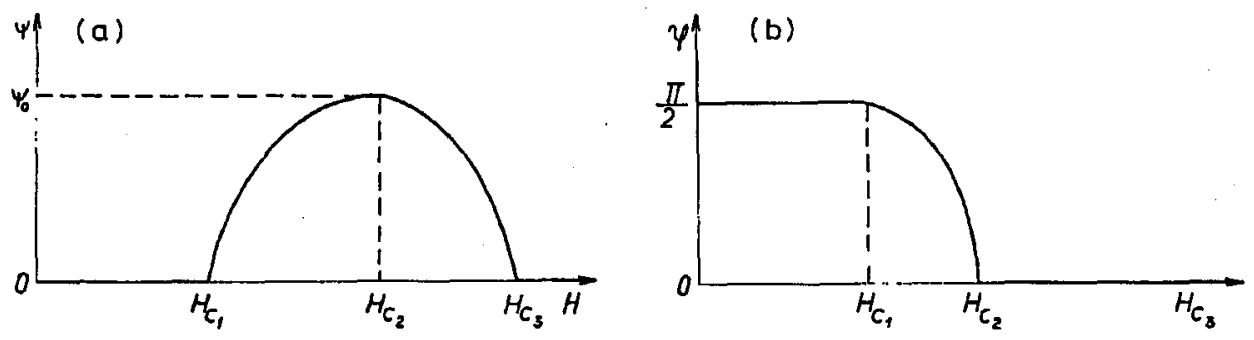

Fig. 4. (a), (b) The dependence of angles of sublattice orientation on the value of the external magnetic field.

$$
\beta_{0}=J_{0}+d_{0}+2 a_{0}+\sqrt{\left(J_{0}+d_{0}+2 a_{0}\right)^{2}+4\left(2 J_{0}-\left|D_{0}\right|\right)^{2}} .
$$

The horizontally cross-hatched area in Fig. 5b determines magnetic states of the system at a pressure $P_{0}$.

As follows from Fig. 5 the set of magnetic states of a strongly anisotropic easy-plane AFM is considerably richer than the one of a weakly anisotropic AFM in which the following phase transitions: QFM-FM1 and FM1-FM2, are realised [5]. 

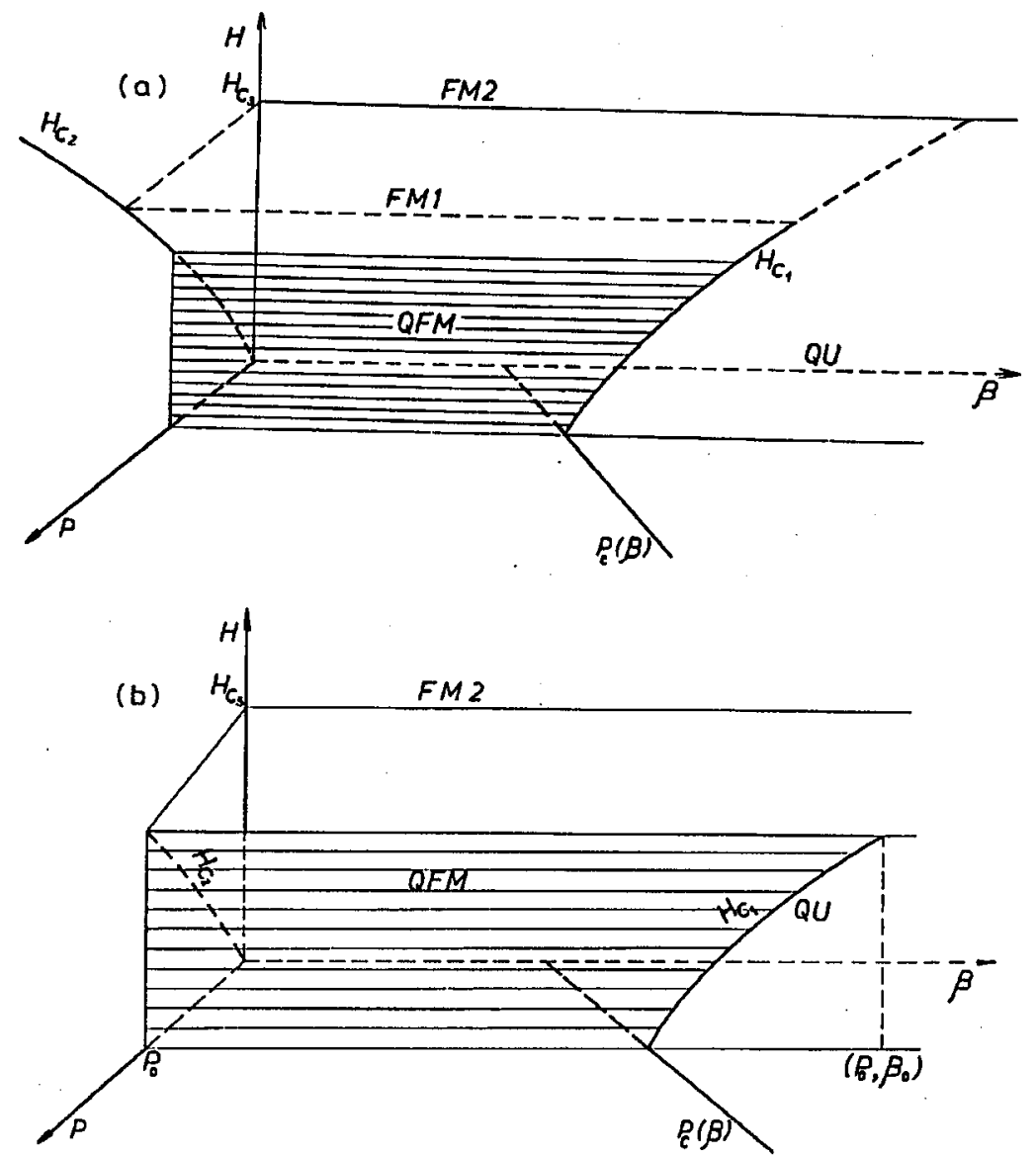

Fig. 5. (a), (b) The phase diagram in coordinates $(P, H, \beta)$ of an easy plane AFM.

\section{Conclusions}

As we have noted above, large one-ion anisotropy and the presence of the external pressure considerably widen the class of possible magnetic states with the easy-plane AFM. One should notice that the external pressure may be interpreted as a presence of certain mechanical boundary conditions [12]. Such an approach is of extreme importance in interpretation of experimental data since taking into account mechanical boundary conditions is a rather complicated and important problem.

The experimental investigation of the phase diagram of the studied system would have been interesting.

\section{References}

[1] I.E. Dickshtein, V.V. Tarasenko, V.G. Shavrov, Zh. Eksp. Teor. Fiz. 67, 816 (1974).

[2] V.G. Borisenko, Yu.V. Pereverzev, Fiz. Nizk. Temp. 11, 730 (1985). 
[3] W.G. Bos, T.O. Khassen, N.J. Poulis, R.L. Carlin, J. Magn. Magn. Mater. 15-18, 464 (1980).

[4] Yu.N. Mitsay, Yu.A. Fridman, Ukr. Fiz. Zh. 35, 459 (1990).

[5] Yu.N. Mitsay, Yu.A. Fridman, Ukr. Fiz. Zh. 35, 758 (1990).

[6] L.D. Landau, E.M. Lifshits, Statistical Physics, Part 1, Nauka, Moskva 1976, p. 583.

[7] R.O. Zaitsev, Zh. Eksp. Teor. Fiz. 63, 207 (1995).

[8] F.P. Onufrieva, Fiz. Tverdogo Tela 26, 3435 (1984).

[9] F. Varret, J. Phys. Chem. Solids 37, 257 (1976).

[10] E.A. Zavadskii, Ph.D. thesis, Makhachkala 1984, p. 131.

[11] A.I. Banshchikov, V.G. Bar'yakhtar, B.A. Ivanov, Yu.N. Mitsay, Sverkhprovod. Fiz. Khim. Tekhn. 4, 433 (1991).

[12] Yu.N. Mitsay, Yu.A. Fridman, G.A. Bairamalieva, Fiz. Tverdogo Tela, 1996, in print. 